\title{
Interethnic DNA methylation difference and its implications in pharmacoepigenetics
}

\author{
Shih-Kai Chu ${ }^{1,2,3}$ \& Hsin-Chou Yang ${ }^{*}, 1,2,4,5,6,7$ \\ ${ }^{1}$ Bioinformatics Program, Taiwan International Graduate Program, Institute of Information Science, Academia Sinica, Taipei 115, \\ Taiwan \\ ${ }^{2}$ Institute of Statistical Science, Academia Sinica, Taipei 115, Taiwan \\ ${ }^{3}$ Institute of Biomedical Informatics, National Yang-Ming University, Taipei 112, Taiwan \\ ${ }^{4}$ Department of Statistics, National Cheng Kung University, Tainan 701, Taiwan \\ ${ }^{5}$ Institute of Statistics, National Tsing Hua University, Hsinchu 300, Taiwan \\ ${ }^{6}$ Instutite of Public Health, National Yang-Ming University, Taipei 112, Taiwan \\ ${ }^{7}$ School of Public Health, National Defense Medical Center, Taipei 114, Taiwan \\ * Author for correspondence: Tel.: +8862 27835611 ext. 113; Fax: +8862 2783 1523; hsinchou@stat.sinica.edu.tw
}

\begin{abstract}
Aim: This is the first systematic study to examine the population differentiation effect of DNA methylation on the treatment response and drug absorption, distribution, metabolism and excretion in multiple tissue types and cancer types. Materials \& methods: We analyzed the whole methylome and transcriptome data of primary tumor tissues of four cancer types (breast, colon, head \& neck and uterine corpus) and lymphoblastoid cell lines for African and European ancestry populations. Results: Ethnicity-associated CpG sites exhibited similar methylation patterns in the two studied populations, but the patterns differed between tumor tissues and lymphoblastoid cell lines. Ethnicity-associated $\mathrm{CpG}$ sites may have triggered gene expression, influenced drug absorption, distribution, metabolism and excretion, and showed tumorspecific patterns of methylation and gene regulation. Conclusion: Ethnicity should be carefully accounted for in future pharmacoepigenetics research.
\end{abstract}

First draft submitted: 24 March 2017; Accepted for publication: 4 July 2017; Published online: 8 September 2017

Keywords: cancer epigenetics $\bullet$ DNA methylation $\bullet$ epipharmacogenomics $\bullet$ population differentiation

DNA methylation, occurring mostly at the C-5 position of cytosine in the CpG dinucleotide, is a fundamental epigenetic mechanism [1]. The main function of DNA methylation is to regulate transcription by recruiting or precluding proteins involved in transcription. The regulation may occur in genomic regions with different $\mathrm{CpG}$ densities, from CpG-rich regions (also known as CpG islands [CGIs]) to single CpG sites [1-4]. For example, 60\% of human genes contain CGIs in their promoter regions, and the hypermethylation of these CGIs suppresses gene expression [4]. Numerous studies have reported that DNA methylation variants are associated with complex traits $[1,5]$ and treatment responses, particularly cancer treatments [6-8]. For example, the hypermethylation of the promoter of the DNA repair gene MGMT can be used to predict an improved clinical response to alkylating agents in glioma patients [9].

Ethnic differences influence the disease risk [10,11] and the treatment outcome [12]. Pharmacogenomics studies have reported that genetic polymorphisms (e.g., single nucleotide polymorphisms [SNPs]) with high population differentiation directly affect the treatment response [13] or are highly related to the absorption, distribution, metabolism and excretion (ADME) of drugs [14,15]. These genetic differences among ethnic populations have motivated additional studies to adjust the recommended drug dosage depending on the population [15]. Ethnic differences are also a vital feature of DNA methylation [16-19]. However, the mechanism through which these differences influence the effect of DNA methylation on the treatment response and the drug ADME remains largely unknown.

With rapid advances in high-throughput methylation profiling techniques in recent years, such as microarrays and next-generation sequencing experiments, abundant methylation data have become available. Therefore, these data present unmet opportunities to detect methylated variants (e.g., CpG sites) or genes associated with the 
drug response to particular disease(s) (MeDR genes). This is the first systematic study to examine the population differentiation effect of DNA methylation on the treatment response and drug ADME. Because methylation patterns are tissue specific [20] and most of the current MeDR genes are related to cancers, we analyzed differential methylation (DM) between African ancestry (AA) and European ancestry (EA) populations in two sources of samples. The first source contained the primary tumor tissues of four cancer types (breast invasive carcinoma [BRCA], colon adenocarcinoma [COAD], head and neck squamous cell carcinoma [HNSC] and uterine corpus endometrial carcinoma [UCEC]) from The Cancer Genome Atlas (TCGA) [21-24], and the second source contained lymphoblastoid cell lines (LCLs) of general populations from the International HapMap Project [17]. We also investigated the relationship between DNA methylation and gene expression and discussed the influence of DNA methylation on the treatment response and drug ADME. The results provide clues about the influence of interethnic differences in DNA methylation on differential gene regulation and pharmacoepigenetics by tissue types.

\section{Materials \& methods}

\section{MeDR genes \& ADME genes}

In this study, to identify MeDR genes, we searched PubMed by using the keywords 'pharmacoepigenetics' or 'pharmacoepigenomics'. Totally, 25 cited studies were obtained. On the basis of these studies, we collected 39 MeDR genes, as shown in Supplementary Table 1. In addition, a set of 298 ADME genes were downloaded from the PharmaADME database (www.pharmaadme.org). According to the human genome, version 19 (hg19), $39 \mathrm{MeDR}$ genes and 294 ADME genes were successfully mapped to the human genome and were included in subsequent analyses (Supplementary Tables $1 \& 2$ ).

\section{Study samples}

We studied 1514 'eligible' samples from four cancer datasets, namely BRCA, COAD, HNSC and UCEC, in TCGA [21-24]. All samples were obtained from the AA or EA population. A sample that met the following requirements was called 'eligible'. First, the sample was a frozen primary tumor tissue. Second, to avoid any effect of a prior treatment on the sample methylation profile, we only included the sample without any history of neoadjuvant treatment. Finally, the sample must have its methylation profile generated by the Infinium HumanMethylation 450 BeadChip [25,26]. In TCGA, only the four aforementioned cancer types had more than 30 eligible samples in each population. The numbers of eligible samples in these four cancer datasets were $84 \mathrm{AA}$ samples and 508 EA samples in the BRCA dataset, 34 AA samples and 206 EA samples in the COAD dataset, 31 AA samples and 321 EA samples in the HNSC dataset and 56 AA samples and 274 EA samples in the UCEC dataset. In this study, AA samples were the samples reported as 'BLACK OR AFRICAN AMERICAN' in the TCGA sample information; EA samples were the samples reported as 'WHITE' in the TCGA sample information.

In addition to the samples from tumor tissues, we studied 133 HapMap LCLs containing 73 AA samples (individuals of Yoruba ethnicity from Ibadan, Nigeria) and 60 EA samples (Caucasian residents of EA from Utah, USA).

\section{DNA methylation data}

For each studied TCGA sample, we downloaded its level 3 methylation profile generated by Infinium HumanMethylation 450 BeadChip with the $\beta$ values of 485,577 CpG sites from the TCGA data portal (Data Type: DNA Methylation). The Infinium HumanMethylation 450 BeadChip is a DNA methylation platform which generates reliable methylation profiles, and its robustness was well validated $[25,26]$. For a specific $\mathrm{CpG}$ site, the $\beta$ value, which was calculated by dividing the methylated signal by the sum of the methylated and unmethylated signals, was considered the methylation level. In the methylation profile of each sample, the TCGA had masked the $\beta$ values of probes with a detection p-value $>0.05$ as not applicable (NA). Moreover, a group of 89,512 CpG sites whose probes contained SNP(s) had been masked as NA in the methylation profiles of all samples across different cancer types. The details of these processing procedures can be found at the TCGA website (http://cancergenome.nih.gov/).

The raw data of the studied HapMap samples were downloaded from NCBI GEO (Accession No.: GSE39672). The methylation profiles were generated by the Infinium HumanMethylation450 BeadChip. We had obtained information on the methylated signals, unmethylated signals and detection $\mathrm{p}$-values in each profile, and the $\beta$ value was calculated to measure the methylation level of each CpG site. 


\section{Gene expression data}

For each studied TCGA sample, we also downloaded their level 3 gene expression profiles from the TCGA data portal (Data Type: RNASeqV2). We analyzed the expression data measured by raw read counts at the gene level generated by RNA sequencing experiments. Raw sequencing reads were aligned against the hg 19 reference genome by using the software Mapsplice [27]. The aligned reads were translated to transcriptome coordinates by using the software UNC Bioinformatics Utilities [28]. The gene abundance was estimated using the software RNA-Seq by Expectation-Maximization [29]. Finally, 20,531 genes were included in each expression profile. The details of data processing from the experiment output to gene expression quantification can be found at the TCGA website (http://cancergenome.nih.gov/).

Among the 133 HapMap samples for which methylation data were obtained, the gene expression profiles of 91 samples were downloaded at EBI ArrayExpress (Accession No.: E-GEUV-1); these data were shared by the Geuvadis RNA sequencing project based on the samples of the 1000 Genomes project [30,31]. These gene expression profiles were generated by RNA sequencing experiments. Raw sequencing reads were aligned against the hg19 reference genome by using Genome Multitool [32]. Transcript quantification was calculated by Flux Capacitor [33]. All transcript quantification results in a gene were then summed up to quantify a gene. Finally, 53,934 genes were included in each expression profile. The details of data processing and quality control are described in a previous study [31].

\section{Data quality control \\ DNA methylation data}

The data quality control (QC) procedure, consisting of per-sample QC, per-probe QC, data normalization and batch effect identification, was performed according to the cancer type (Supplementary Figure 1). In the BRCA dataset, we restricted our analysis to samples from female patients; seven samples from male BRCA patients were removed before the data QC procedure. First, in the per-sample QC step, samples whose missing rate across all probes exceeded 5\% were removed. Samples located outside the confidence region in the principle component scatter plots made on the basis of the sex chromosome data were also removed. In the per-probe QC step, probes whose missing rate across all samples was more than 0.05 were removed. Second, probes that had missing rates in the $\mathrm{AA}$ and EA populations that were significantly different based on the Bonferroni corrected p-value of a two-sample proportion test were removed. After filtering out problematic probes, the data of CpG sites on autosomes were extracted for the subsequent analysis. Third, in the data normalization step, to correct for the Infinium I/II type bias within each array, the Beta-Mixture Quantile Normalization (BMIQ) method [34] was applied to each sample. Finally, in the batch effect identification step, the 'known' batches (biospecimen shipping in TCGA) were adjusted for by using the software ComBat [35], and the 'unwanted' factors and batches were inferred and then adjusted for by using surrogate variable analysis (SVA) [36]. The aforementioned QC procedures were also applied to the HapMap dataset (Supplementary Figure 1). Here, the known batch was the batches of array hybridization.

\section{Gene expression data}

The QC procedure consisted of per-gene QC and batch effect identification (Supplementary Figure 2). Both TCGA and HapMap datasets were examined using the same procedure. In the per-gene QC step, the genes with a zero read count across all samples were removed. In the batch effect identification, SVA was performed on the remaining genes. ComBat was not adopted here because this method was not recommended for analyzing read counts that are integers.

\section{Statistical analyses}

Summary statistics of methylation difference between two ethnic populations

To measure the global difference in the methylation level in each dataset, we first defined $D_{i}=\bar{x}_{i}^{A A}-\bar{x}_{i}^{E A}$ for probe $\mathrm{i}$, where $\overline{\mathrm{x}}_{\mathrm{i}}^{\mathrm{AA}}$ and $\overline{\mathrm{x}}_{\mathrm{i}}^{\mathrm{EA}}$ indicate the average BMIQ normalized $\beta$ value of probe $\mathrm{i}$ in the AA population and EA population, respectively. The summary statistic $\bar{D}$ indicates the average of $D_{i}$ across probes in the whole methylome, and $\overline{\mathrm{D}_{\mathrm{abs}}}$ indicates the average of $\left|\mathrm{D}_{\mathrm{i}}\right|$ across probes in the whole methylome. 


\section{$D M$ analysis}

Three linear regression models for different batch effect correction scenarios were applied to test the association between the methylation level of each probe and the sample ethnicity. For more statistical validity in DM and other statistical analyses, the $\beta$ value was logit-transformed in the following models [37]:

- $\left(\right.$ Model A) $\mathrm{M}_{\mathrm{ij}}=\alpha_{\mathrm{i}}+\beta_{\mathrm{i}} \mathrm{E}_{\mathrm{j}}+\sum_{\mathrm{k}=1}^{\mathrm{K}} \gamma_{\mathrm{ki}} \mathrm{G}_{\mathrm{kj}}+\varepsilon_{\mathrm{ij}}$

- (Model B) $\mathrm{M}_{\mathrm{ij}}=\alpha_{\mathrm{i}}+\beta_{\mathrm{i}} \mathrm{E}_{\mathrm{j}}+\sum_{\mathrm{k}=1}^{\mathrm{K}} \gamma_{\mathrm{ki}} \mathrm{G}_{\mathrm{kj}}+\sum_{\mathrm{l}=1}^{\mathrm{L}} \delta_{\mathrm{li}} \mathrm{S}_{\mathrm{lj}}+\varepsilon_{\mathrm{ij}}$

- (Model C) $\mathrm{M}_{\mathrm{ij}}^{*}=\alpha_{\mathrm{i}}+\beta_{\mathrm{i}} \mathrm{E}_{\mathrm{j}}+\sum_{\mathrm{k}=1}^{\mathrm{K}} \gamma_{\mathrm{ki}} \mathrm{G}_{\mathrm{kj}}+\varepsilon_{\mathrm{ij}}$

where

$M_{i j}$ is the logit-transformed $\beta$ value for probe $i$ of sample $j, i=1, \ldots, m, j=1, \ldots, n$ ( $m$ is the number of probes in the whole methylome; $\mathrm{n}$ is the total number of samples in the AA and EA populations).

$\mathrm{M}_{\mathrm{ij}}^{*}$ is the logit-transformed $\beta$ value that is batch adjusted using ComBat for probe $\mathrm{i}$ of sample $\mathrm{j}$.

$E_{j}$ is the ethnicity information (i.e., an indicator of the AA or EA population) of sample $j$.

$\mathrm{G}_{\mathrm{kj}}$ is the kth covariate value for adjustment of sample $\mathrm{j}, \mathrm{k}=1, \ldots, \mathrm{K}$ ( $\mathrm{K}$ is the number of covariates).

$\mathrm{S}_{\mathrm{lj}}$ is the lth surrogate variable value of sample $\mathrm{j}, \mathrm{l}=1, \ldots, \mathrm{L}$ ( $\mathrm{L}$ is the number of surrogate variables).

$\alpha_{\mathrm{i}}, \beta_{\mathrm{i}}, \gamma_{\mathrm{ki}}$ and $\delta_{\mathrm{li}}$ are the regression coefficients.

$\epsilon_{\mathrm{ij}}$ is the random error for probe $\mathrm{i}$ of sample $\mathrm{j}$.

In each model, ethnicity information was the primary variable of interest. Moreover, the three covariates of sex, tumor staging and age were considered in the analyses of the cancer datasets, except for sex, which was not included in the analyses of the BRCA and UCEC datasets because all analyzed samples were obtained from female patients. One covariate of sex was included in the analysis of the HapMap dataset. Regarding batch effect adjustment, in model A, no batch effect was assumed. In model B, the surrogate variables estimated using SVA [36] were adjusted for in order to remove the variation of all unwanted factors. In model $\mathrm{C}$, we removed the potential batch effect by using ComBat [35]. For each probe under a model, we assessed the significance of $\beta$ by using the $t$-test, and the resultant p-value was further adjusted by Bonferroni correction [38] for addressing the multiple testing problems. Probes were considered as differentially methylated CpG sites if their Bonferroni corrected p-values were $<0.05$.

We used all three models in the analyses of the cancer datasets, whereas only models B and C were used in the analysis of the HapMap dataset because of the obvious batch effect. In each dataset, the CpG sites that were differentially methylated in at least one of the three models were considered as E-CpGs.

\section{Relationship between DNA methylation \& gene expression}

Given an E-CpG from DM analysis, the flowchart to establish the relationship between its methylation level and the expression of the corresponding gene is shown in Supplementary Figure 3. Briefly, we first calculated the correlation coefficients between E-CpG methylation and the corresponding gene expression (CorMG) in each population. If a significant correlation was found in at least one population, we then verified whether the gene was differentially expressed between two populations in a differential expression (DE) analysis. If a significant DE was found in at least one model, we finally verified whether a straightforward relationship exists between E-CpG methylation and the corresponding gene expression. Each step described above is described in detail in the Supplementary Methods.

\section{Results}

\section{Data QC \& global DNA methylation patterns}

A series of data QC procedures for methylation data were performed for each of the BRCA, COAD, HNSC, UCEC and HapMap datasets before DM analysis (as described in the 'Materials \& methods' section; Supplementary Figure 1). In the per-sample QC step, no samples were removed because the missing rate exceeded $5 \%$ across all probes, and $0(12,2,0$ and 0$)$ samples were excluded from the BRCA (COAD, HNSC, UCEC and HapMap) dataset because they were outliers in the corresponding self-reported sex group (Supplementary Figure 4). In the per-probe QC step, 11,576 (11,699, 11,048, 10,661 and 16,655) probes were filtered out because they were identified as problematic probes or interrogated on sex chromosomes. Each methylation profile was then normalized using the BMIQ method [34], followed by a batch effect adjustment procedure. The demographic characteristics (ethnicity, 


\begin{tabular}{|c|c|c|c|c|c|c|}
\hline Datasets & Probes & \# CpG & $\overline{\mathrm{D}}$ & $\operatorname{sd}(D)$ & $\overline{\mathrm{D}_{\mathrm{abs}}}$ & $s d\left(D_{\text {abs }}\right)$ \\
\hline \multirow[t]{2}{*}{ BRCA } & All probes & 384,489 & -0.0015 & 0.0273 & 0.0170 & 0.0213 \\
\hline & E-CpGs & 2638 & -0.0087 & 0.0749 & 0.0587 & 0.0473 \\
\hline \multirow[t]{2}{*}{ COAD } & All probes & 384,366 & -0.0040 & 0.0231 & 0.0148 & 0.0182 \\
\hline & $\mathrm{E}-\mathrm{CpGs}$ & 373 & -0.0118 & 0.1058 & 0.0795 & 0.0707 \\
\hline \multirow[t]{2}{*}{ HNSC } & All probes & 385,017 & 0.0003 & 0.0281 & 0.0179 & 0.0217 \\
\hline & E-CpGs & 307 & 0.0311 & 0.0962 & 0.0791 & 0.0629 \\
\hline \multirow[t]{2}{*}{ UCEC } & All probes & 385,404 & -0.0028 & 0.0280 & 0.0173 & 0.0223 \\
\hline & E-CpGs & 721 & -0.0052 & 0.0903 & 0.0668 & 0.0609 \\
\hline \multirow[t]{2}{*}{ HapMap } & All probes & 379,410 & 0.0208 & 0.0504 & 0.0329 & 0.0435 \\
\hline & $\mathrm{E}-\mathrm{CpGs}$ & 20,287 & 0.1364 & 0.0560 & 0.1394 & 0.0480 \\
\hline
\end{tabular}

sex and age) and clinical characteristics (cancer stage) of the samples that passed the aforementioned QC procedures in each dataset are summarized in Supplementary Table 3.

Regarding the global DNA methylation patterns, for all post-QC CpG sites in the cancer and HapMap datasets, no significant difference ( $\bar{D} \approx 0$ ) was observed in the average $\beta$ values of samples between the AA and EA populations (Table 1). Several DNA methylation patterns were observed in the different regions of a gene in all datasets (Figure 1A-N for the BRCA and HapMap datasets; Supplementary Figure 5A-U for the COAD, HNSC and UCEC datasets; Supplementary Table 4A-F for the comparative tests and the resultant p-values). First, CpG sites in CGIs had a lower median methylation level than did those in non-CGIs in the human methylome (Figure 1A \& H; Supplementary Figure 5A, H \& O; Supplementary Table 4A). Second, CpG sites closer to transcription start sites (TSSs) showed a lower median methylation level (Figure 1B, C, I \& J; Supplementary Figure 5B, C, I, J, P \& Q) than did those in the whole methylome (Figure 1A,H; Supplementary Figure 5A, $\mathrm{H} \& \mathrm{O}$; Supplementary Table 4B), and most CpG sites in CGIs were not methylated (Figure 1B, C, I \& J; Supplementary Figure 5B, C, I, J, P \& Q; Supplementary Table 4C). Third, CpG sites in 5'-untranslated and the regions of the first exon had a lower median methylation level (Figure 1D, E, K \& I; Supplementary Figure 5D, E, K, L, R \& S) than did those in the whole methylome (Figure 1A,H; Supplementary Figure 5A, H \& O; Supplementary Table 4D), and most CpG sites in CGIs were not methylated (Figure 1D, E, K L; Supplementary Figure 5D, E, K, L, R \& S; Supplementary Table 4E). Fourth, CpG sites within gene bodies and 3'-untranslated regions (Figure 1F, G, M \& N; Supplementary Figure 5F, G, M, N, T \& U) had a higher median methylation level than did those in the whole methylome (Figure 1A \& H; Supplementary Figure 5A, H \& O; Supplementary Table 4F). Finally, the aforementioned patterns can be found in both AA and EA populations and in all cancer and HapMap datasets.

DNA methylation patterns of ethnicity-associated CpG sites

DM analysis in post-QC datasets identified 2638 (373, 307, 721 and 20,287) ethnicity-associated CpG sites (E-CpGs) in the BRCA (COAD, HNSC, UCEC and HapMap) dataset. For E-CpGs, the difference in the $\beta$ value between the AA and EA populations ( $\overline{\mathrm{D}_{\mathrm{abs}}}$ in notation) in the five studied datasets ranged from 0.0587 to 0.1394 (Table 1). $\overline{\mathrm{D}}$ was close to 0 and generally smaller than $\overline{\mathrm{D}_{\text {abs }}}$ in the cancer datasets but was close to $\overline{\mathrm{D}_{\text {abs }}}$ in the HapMap dataset. This result indicates that the methylation levels of most E-CpGs in the AA population were similar to those in the EA population in the cancer datasets but were higher than those in the EA population in the HapMap dataset.

When we focused on E-CpGs, two distinct methylation patterns were observed between the cancer datasets and the HapMap dataset (Figure 2A-N for the BRCA and HapMap datasets; Supplementary Figure 6A-U for the COAD, HNSC and UCEC datasets; Supplementary Table 4G,H for the comparative tests and the resultant pvalues). First, in the cancer datasets, the global methylation patterns of E-CpGs were similar to those of overall CpGs described in the previous section (Figure 2A-G vs Figure 1A-G; Supplementary Figure 6A-G vs Supplementary Figure 5A-G; Supplementary Figure 6H-N vs Supplementary Figure 5H-N; Supplementary Figure 6O-U vs 


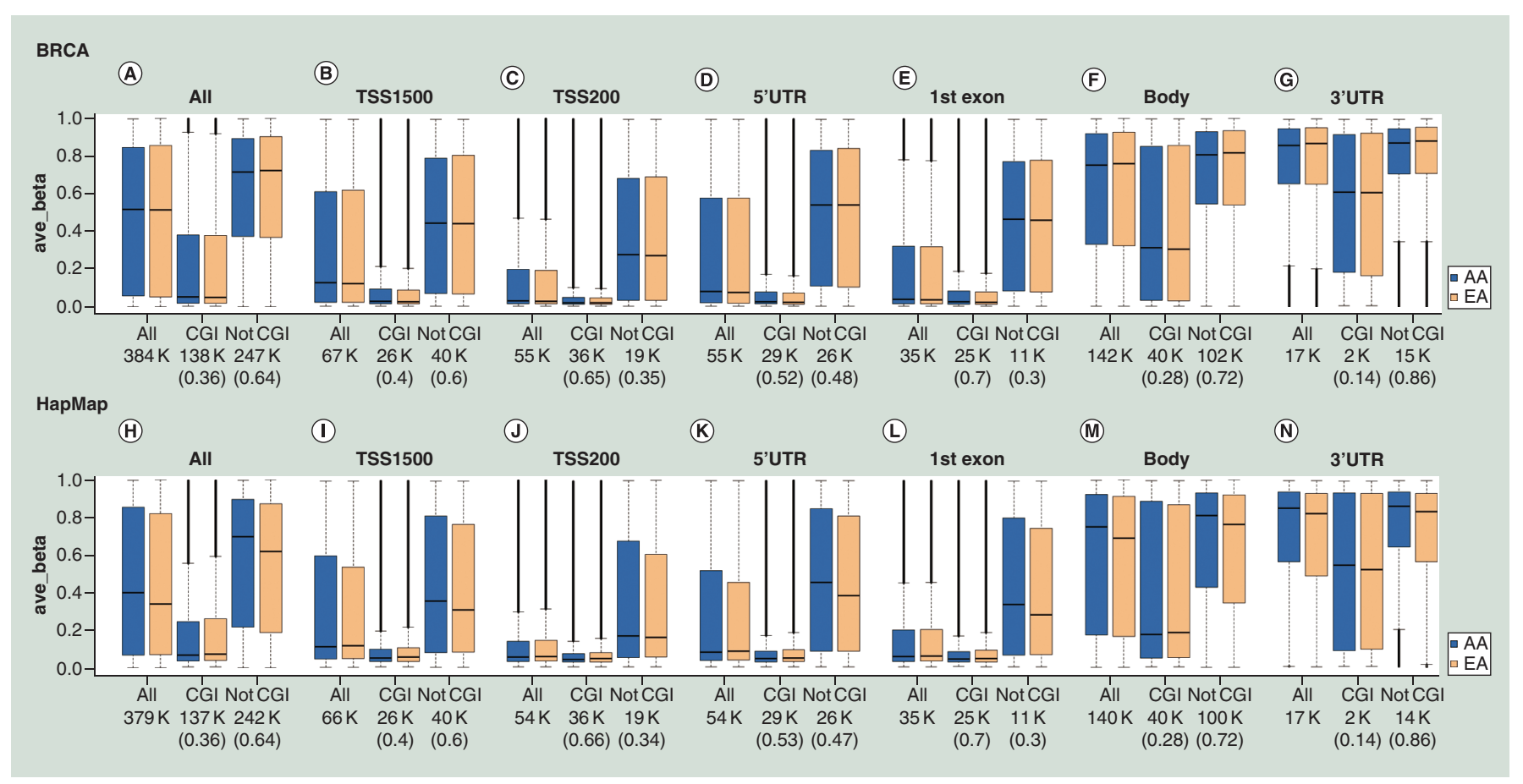

Figure 1. Distribution of DNA methylation patterns of the $\mathrm{CpG}$ sites in African ancestry (blue) and European ancestry (peach) populations in BRCA and HapMap datasets.

The results of the BRCA dataset are shown in (A-G) and the results of the HapMap dataset are shown in (H-N). In the Tukey boxplots, the lower and upper whiskers are defined by subtracting 1.5-times interquartile range from the first quartile and adding 1.5-times interquartile range from the third quartile, respectively. (A \& H) 'All' displays the distribution of all CpG sites across the methylome. CpG sites in 'All' are divided by different gene regions and further by the relationship to the CGI; (B \& I) 'TSS1500': the region between 200 and 1500 bp upstream of a TSS. (C \& J) 'TSS200': the region within 200 bp upstream of a TSS; (D \& K) '5'UTR': the 5'-untranslated region; (E \& I) '1stExon': exon 1 region; (F \& M) 'Body': gene body; (G \& N) '3'UTR': 3'-untranslated region. Subgroup 'CGI' indicates CpG sites located in CpG islands and subgroup 'notCGI' indicates CpG sites not located in CGIs. The number of CpG sites in each group and the proportion of sites in CGI (in brackets) are listed under the plot.

AA: African ancestry; EA: European ancestry; CGI: CpG island; TSS: Transcription start site.

Supplementary Figure 5O-U; Supplementary Table 4G). However, in the HapMap dataset, low methylation in promoter regions (TSS1500 and TSS200) and high methylation in the gene body and $3^{\prime}$-untranslated regions in overall CpGs became less obvious in E-CpGs (Figure $2 \mathrm{H}-\mathrm{N}$ vs Figure $1 \mathrm{H}-\mathrm{N}$; Supplementary Table $4 \mathrm{H}$ ). Second, in each cancer dataset, the proportion of E-CpGs in CGIs was generally higher than that of CpG sites in CGIs relative to all analyzed CpG sites (e.g., $0.4>0.36$ in the BRCA dataset, $\mathrm{p}=2.024 \times 10^{-5}$ ). However, in the HapMap dataset, the proportion of E-CpGs in CGIs was markedly lower than that of CpG sites in CGIs relative to all analyzed CpG sites (e.g., $0.07<0.36, \mathrm{p}<2.2 \times 10^{-16}$ ).

Interethnic methylation differences in MeDR genes

Our PubMed search found that 22 (5, 2 and 0) MeDR genes were associated with breast cancer (colon cancer, head and neck cancer and endometrial cancer) (Supplementary Table 1). After mapping 2638 (373, 307 and 721) E-CpGs in the BRCA (COAD, HNSC and UCEC) dataset to the MeDR genes of the respective cancer only two crucial E-CpGs in the BRCA dataset were located on the MeDR genes for breast cancer; they were $\operatorname{cg} 16919093$ on the MeDR gene BRCA1 and cg10554436 on the MeDR gene FOXL2 (Table 2). No E-CpGs in the COAD, HNSC and UCEC datasets were located on MeDR genes of the respective cancers.

The E-CpG cg16919093 was located in an intron region of the MeDR gene BRCA1. The E-CpG cg16919093 exhibited a significantly lower average $\beta$ value in the AA population than in the EA population in the BRCA dataset $\left(\mathrm{p}_{\text {Model A }}=1.07 \times 10^{-8}\right.$, $\mathrm{p}_{\text {Model B }}=1.17 \times 10^{-15}$, pModel $\mathrm{C}=3.35 \times 10^{-15}$, Table $2 \mathrm{~A}$; see Materials $\&$ methods for details). The lower methylation levels of $\mathrm{CpG}$ sites near $B R C A 1$ were associated with improved clinical outcomes in node-positive, estrogen receptor-positive, HER-2-negative breast cancer patients receiving adjuvant 


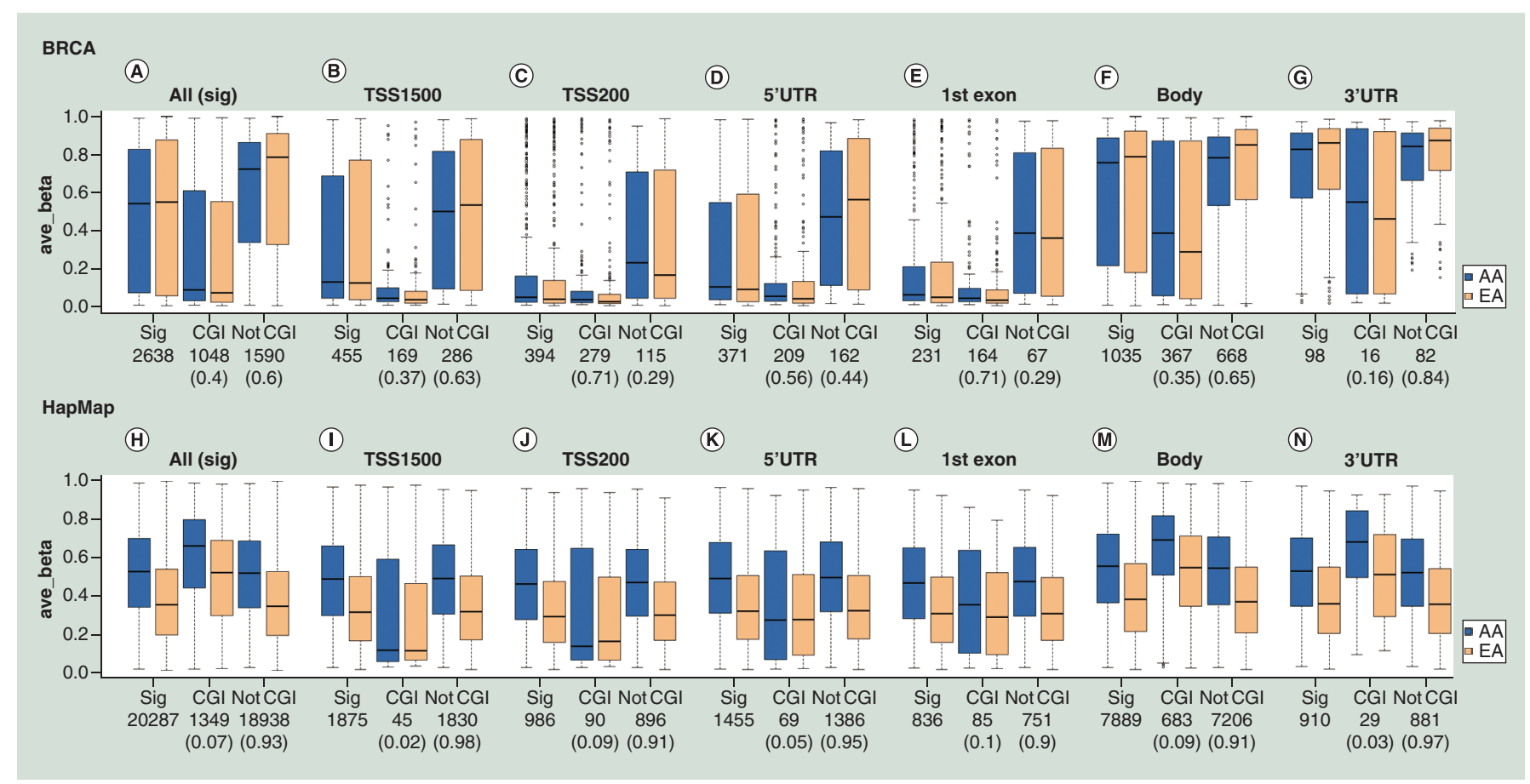

Figure 2. Distribution of DNA methylation patterns of the E-CpG sites in Africa ancestry (blue) and European ancestry (peach) populations in BRCA and HapMap datasets.

The results of the BRCA dataset are shown in (A-G) and the results of the HapMap dataset are shown in (H-N). In the Tukey boxplots, the lower and upper whiskers are defined by subtracting 1.5-times interquartile range from the first quartile and adding 1.5-times interquartile range from the third quartile, respectively. (A \& H) 'All (sig)' displays the distribution of all E-CpG sites across the methylome. E-CPG sites in 'All (sig)' are divided by different gene regions and further by the relationship to the CGI; (B \& I) 'TSS1500': the region between 200 and 1500 bp upstream of a TSS. (C \& J) 'TSS200': the region within 200 bp upstream of a TSS; (D \& K) '5'UTR': the 5'-untranslated region; (E \& L) '1stExon': exon 1 region; (F \& M) 'Body': gene body; (G \& N) '3'UTR': 3'-untranslated region. Subgroup 'CGI' indicates E-CpG sites located in CGls and subgroup 'notCGI' indicates E-CpG sites not located in CGIs. The number of E-CpG sites in each group and the proportion of sites in CGI (in brackets) are listed under the plot.

AA: African ancestry; CGI: CpG island; EA: European ancestry; TSS: Transcription start site.

\begin{tabular}{|c|c|c|c|c|c|c|c|c|c|}
\hline \multirow{2}{*}{$\begin{array}{l}\text { Gene } \\
\text { symbol }\end{array}$} & \multirow{2}{*}{$\begin{array}{l}\text { Drug name (its MeDR } \\
\text { effect) }\end{array}$} & \multirow[t]{2}{*}{ E-CpG id } & \multicolumn{3}{|c|}{ DM analysis } & \multicolumn{4}{|c|}{ CorMG analysis } \\
\hline & & & $\begin{array}{l}\text { B_AA (1: ave, } \\
\text { 2: sd) }\end{array}$ & $\begin{array}{l}\text { B_EA (1: ave, } \\
\text { 2: sd) }\end{array}$ & $\begin{array}{l}\text { DM p-values } \\
\text { (1: Model A, } \\
\text { 2: Model B, } \\
\text { 3: Model C) }\end{array}$ & $\begin{array}{l}\text { All (1: CorMG, } \\
\text { 2: p-value) }\end{array}$ & $\begin{array}{l}\text { AA (1: CorMG, } \\
\text { 2: p-value) }\end{array}$ & $\begin{array}{l}\text { EA (1: CorMG, } \\
\text { 2: p-value) }\end{array}$ & $\begin{array}{l}\text { p-value } \\
\text { (CorMG_AA vs } \\
\text { CorMG_EA) }\end{array}$ \\
\hline$B R C A 1$ & $\begin{array}{l}\text { - Anthracycline (lower } \\
\text { Me. to better response) } \\
\text { PARP inhibitors (hyper } \\
\text { Me. to higher } \\
\text { sensitivity) }\end{array}$ & cg16919093 & $\begin{array}{ll}1 & 0.8831 \\
2 & 0.1200\end{array}$ & $\begin{array}{ll}1 & 0.9442 \\
2 & 0.0542\end{array}$ & $\begin{array}{ll}1 & 1.07 \times 10^{-8} \\
2 & 1.17 \times 10^{-15} \\
3 & 3.35 \times 10^{-15}\end{array}$ & $\begin{array}{ll}1 & 0.1440 \\
2 & 4.83 \times 10^{-4}\end{array}$ & $\begin{array}{ll}1 & -0.0956 \\
2 & 0.4010\end{array}$ & $\begin{array}{ll}1 & 0.1740 \\
2 & 8.57 \times 10^{-5}\end{array}$ & 0.0232 \\
\hline FOXL2 & $\begin{array}{l}\text { Anthracycline (lower } \\
\text { Me. to better response) }\end{array}$ & cg10554436 & $\begin{array}{ll}1 & 0.8395 \\
2 & 0.1715\end{array}$ & $\begin{array}{ll}1 & 0.8909 \\
2 & 0.1194\end{array}$ & $\begin{array}{ll}1 & 0.0202 \\
2 & 1.23 \times 10^{-6} \\
3 & 1.29 \times 10^{-7}\end{array}$ & $\begin{array}{ll}1 & 0.0197 \\
2 & 0.6330\end{array}$ & $\begin{array}{ll}1 & 0.1035 \\
2 & 0.3490\end{array}$ & $\begin{array}{ll}1 & 0.0245 \\
2 & 0.5830\end{array}$ & 0.5080 \\
\hline
\end{tabular}

anthracycline-based chemotherapy [39]. Moreover, CGI hypermethylation in BRCA1 predicted sensitivity in breast cancer patients to the PARP inhibitors [40].

We calculated the correlation coefficient CorMG (see Materials \& methods for details) between the CpG cg16919093 methylation and BRCA1 gene expression. The result showed that CorMG in the AA population was significantly lower than that in the EA population $(\mathrm{p}=0.0232$, Table $2 \mathrm{~A})$. CorMG was not significantly 


\begin{tabular}{|c|c|c|c|c|c|c|c|c|c|}
\hline \multirow[t]{2}{*}{ Datasets } & \multirow{2}{*}{$\begin{array}{l}\text { Gene symbol } \\
\text { (ADME class) }\end{array}$} & \multirow[t]{2}{*}{ E-CpG id } & \multicolumn{3}{|c|}{ DM analysis } & \multicolumn{4}{|c|}{ CorMG analysis } \\
\hline & & & $\begin{array}{l}\text { B_AA (1: ave, } \\
\text { 2: sd) }\end{array}$ & $\begin{array}{l}\text { B_EA (1: ave, } \\
\text { 2: sd) }\end{array}$ & $\begin{array}{l}\text { DM p-values } \\
\text { (1: Model A, } \\
\text { 2: Model B, } \\
\text { 3: Model C) }\end{array}$ & $\begin{array}{l}\text { All (1: CorMG, } \\
\text { 2: p-value) }\end{array}$ & $\begin{array}{l}\text { AA (1: CorMG, } \\
\text { 2: p-value) }\end{array}$ & $\begin{array}{l}\text { EA (1: CorMG, } \\
\text { 2: p-value) }\end{array}$ & $\begin{array}{l}\text { p-value } \\
\text { (CorMG_AA vs } \\
\text { CorMG_EA) }\end{array}$ \\
\hline \multirow[t]{4}{*}{$B R C A$} & DHRS7 (Phase I) & cg23283614 & $\begin{array}{ll}1 & 0.5206 \\
2 & 0.2510\end{array}$ & $\begin{array}{ll}1 & 0.3139 \\
2 & 0.2195\end{array}$ & $\begin{array}{l}14.47 \times 10^{-13} \\
29.86 \times 10^{-15} \\
38.32 \times 10^{-13}\end{array}$ & $\begin{array}{ll}1 & -0.0712 \\
2 & 0.0839\end{array}$ & $\begin{array}{ll}1 & -0.0256 \\
2 & 0.8170\end{array}$ & $\begin{array}{ll}1 & -0.0484 \\
2 & 0.2770\end{array}$ & 0.8480 \\
\hline & & $\operatorname{cg} 24461412$ & $\begin{array}{ll}1 & 0.0356 \\
2 & 0.0297\end{array}$ & $\begin{array}{ll}1 & 0.0240 \\
2 & 0.0098\end{array}$ & $\begin{array}{ll}1 & 8.34 \times 10^{-10} \\
2 & 4.62 \times 10^{-10} \\
3 & 6.73 \times 10^{-13}\end{array}$ & $\begin{array}{ll}1 & -0.1978 \\
2 & 1.32 \times 10^{-6}\end{array}$ & $\begin{array}{ll}1 & -0.1795 \\
2 & 0.1020\end{array}$ & $\begin{array}{l}1-0.1809 \\
24.29 \times 10^{-5}\end{array}$ & 0.9900 \\
\hline & & cg00846580 & $\begin{array}{ll}1 & 0.0719 \\
2 & 0.0208\end{array}$ & $\begin{array}{ll}1 & 0.0506 \\
2 & 0.0119\end{array}$ & $\begin{array}{ll}1 & 5.22 \times 10^{-33} \\
2 & 2.98 \times 10^{-29} \\
3 & 1.13 \times 10^{-40}\end{array}$ & $\begin{array}{l}1-0.2763 \\
21.00 \times 10^{-11}\end{array}$ & $\begin{array}{l}1-0.4317 \\
24.90 \times 10^{-5}\end{array}$ & $\begin{array}{l}1-0.2445 \\
22.71 \times 10^{-8}\end{array}$ & 0.0760 \\
\hline & & cg18906360 & $\begin{array}{ll}1 & 0.7597 \\
2 & 0.1647\end{array}$ & $\begin{array}{ll}1 & 0.6931 \\
2 & 0.1691\end{array}$ & $\begin{array}{ll}1 & 7.68 \times 10^{-04} \\
2 & 2.13 \times 10^{-12} \\
3 & 1.02 \times 10^{-03}\end{array}$ & $\begin{array}{ll}1 & -0.0754 \\
2 & 0.0671\end{array}$ & $\begin{array}{ll}1 & -0.0316 \\
2 & 0.7750\end{array}$ & $\begin{array}{ll}1 & -0.0623 \\
2 & 0.1620\end{array}$ & 0.7970 \\
\hline \multirow[t]{4}{*}{$B R C A$} & DHRS4 (Phase I) & cg01878807 & $\begin{array}{ll}1 & 0.4532 \\
2 & 0.2191\end{array}$ & $\begin{array}{ll}1 & 0.2449 \\
2 & 0.1474\end{array}$ & $\begin{array}{ll}1 & 1.93 \times 10^{-21} \\
2 & 8.70 \times 10^{-27} \\
3 & 5.14 \times 10^{-21}\end{array}$ & $\begin{array}{l}1-0.2337 \\
29.95 \times 10^{-9}\end{array}$ & $\begin{array}{ll}1 & -0.2904 \\
2 & 0.0076\end{array}$ & $\begin{array}{l}1-0.2687 \\
29.16 \times 10^{-10}\end{array}$ & 0.8440 \\
\hline & & cg01937701 & $\begin{array}{ll}1 & 0.0563 \\
2 & 0.0931\end{array}$ & $\begin{array}{ll}1 & 0.0258 \\
2 & 0.0522\end{array}$ & $\begin{array}{ll}1 & 7.89 \times 10^{-10} \\
2 & 8.71 \times 10^{-09} \\
3 & 1.21 \times 10^{-07}\end{array}$ & $\begin{array}{ll}1 & -0.1051 \\
2 & 0.0106\end{array}$ & $\begin{array}{ll}1 & -0.0516 \\
2 & 0.6410\end{array}$ & $\begin{array}{ll}1 & -0.1224 \\
2 & 0.0058\end{array}$ & 0.5510 \\
\hline & & cg05171021 & $\begin{array}{ll}1 & 0.1112 \\
2 & 0.1525\end{array}$ & $\begin{array}{ll}1 & 0.0263 \\
2 & 0.0496\end{array}$ & $\begin{array}{ll}1 & 7.82 \times 10^{-25} \\
2 & 6.12 \times 10^{-28} \\
3 & 1.47 \times 10^{-26}\end{array}$ & $\begin{array}{ll}1 & -0.0908 \\
2 & 0.0282\end{array}$ & $\begin{array}{ll}1 & -0.1829 \\
2 & 0.1070\end{array}$ & $\begin{array}{ll}1 & -0.1326 \\
2 & 0.0029\end{array}$ & 0.6660 \\
\hline & & cg18981338 & $\begin{array}{ll}1 & 0.2930 \\
2 & 0.1600\end{array}$ & $\begin{array}{ll}1 & 0.1866 \\
2 & 0.1256\end{array}$ & $\begin{array}{ll}1 & 1.76 \times 10^{-09} \\
2 & 2.77 \times 10^{-19} \\
3 & 1.25 \times 10^{-08}\end{array}$ & $\begin{array}{ll}1 & -0.2741 \\
2 & 1.47 \times 10^{-11}\end{array}$ & $\begin{array}{l}1-0.4296 \\
25.37 \times 10^{-5}\end{array}$ & $\begin{array}{ll}1 & -0.2808 \\
2 & 1.46 \times 10^{-10}\end{array}$ & 0.1540 \\
\hline
\end{tabular}

different from 0 in the AA population $(\mathrm{p}=0.401$, Table $2 \mathrm{~A})$; however, CorMG was significantly positive in the EA population $\left(\mathrm{p}=8.57 \times 10^{-5}\right.$, Table $\left.2 \mathrm{~A}\right)$.

Another E-CpG in the BRCA dataset was cg10554436 in the promoter region (outside CGI) of FOXL2. Its average $\beta$ value in the AA population was significantly lower than that in the EA population ( $\mathrm{p}_{\text {Model } \mathrm{A}}=0.0202$, pModel $\mathrm{B}=1.23 \times 10^{-6}$, p podel C $=1.29 \times 10^{-7}$, Table $2 \mathrm{~B}$ ). The lower methylation levels of CpG sites near FOXL2 were associated with improved clinical outcomes in node-positive, estrogen receptor-positive, HER-2-negative breast cancer patients receiving adjuvant anthracycline-based chemotherapy [39]. We also calculated the CorMG between the E-CpG cg10554436 methylation and FOXL2 gene expression, but CorMG was not significant, irrespective of ethnicity ( $\mathrm{p}=0.349$ in $\mathrm{AA}$ and $\mathrm{p}=0.583$ in EA, Table $2 \mathrm{~B}$ ).

Interethnic methylation differences in ADME genes

Of all CpG sites included in the BRCA (COAD, HNSC, UCEC and HapMap) DM analysis, 4227 (4224, 4228, 4230 and 4192) $\mathrm{CpG}$ sites were located in or close to the studied ADME genes. Of the CpG sites located close to the ADME genes, 35 (6, 8, 9 and 227) CpG sites were identified as E-CpGs located in 24 (5, 6, 7 and 109) ADME genes (Supplementary Table 5) in the BRCA (COAD, HNSC, UCEC and HapMap) dataset.

We identified two crucial E-CpGs (cg23283614 on DHRS7 and cg01878807 on DHRS4) that exhibited a large interethnic difference in the $\beta$ value, and these $\mathrm{E}-\mathrm{CpG}$ sere located in the ADME gene regions with multiple E-CpGs from the BRCA dataset (Table 3 \& Supplementary Table 5B). The E-CpG cg23283614 was located in the promoter region of gene DHRS7, which is involved in Phase I metabolism, and its average $\beta$ value in the AA population was significantly higher than that in the EA population by $\sim 0.2$ ( p Model A $=4.47 \times 10^{-13}$, 


\begin{tabular}{|c|c|c|c|c|c|c|c|c|c|c|}
\hline \multirow{3}{*}{$\begin{array}{l}\text { Gene } \\
\text { symbol }\end{array}$} & \multirow{3}{*}{$\begin{array}{l}\text { ADME class } \\
\text { Phase I }\end{array}$} & \multirow{3}{*}{$\begin{array}{l}\text { Datasets } \\
\text { COAD }\end{array}$} & \multirow{3}{*}{$\begin{array}{l}\text { E-CpG id } \\
\text { cg00846580 }\end{array}$} & \multirow{3}{*}{\multicolumn{2}{|c|}{\begin{tabular}{l}
\multicolumn{2}{c}{ DM analysis ${ }^{\dagger}$} \\
$\beta$ _AA vs $\beta$ _EA \\
$+\quad(0.0161)$
\end{tabular}}} & \multicolumn{4}{|c|}{ CorMG analysis ${ }^{\ddagger}$} & \multirow{2}{*}{$\begin{array}{c}\text { DE analysis } \\
\text { Logcpm_AA vs Logcpm_EA }\end{array}$} \\
\hline & & & & & & \multicolumn{2}{|c|}{ CorMG_AA } & \multicolumn{2}{|c|}{ CorMG_EA } & \\
\hline & & & & & & nsig & $(-0.1936)$ & - & $(-0.3084)$ & $-\quad(-0.1883)$ \\
\hline DHRS4 & Phase I & UCEC & cg01878807 & + & $(0.1583)$ & - & $(-0.3530)$ & nsig & $(-0.0623)$ & $(-0.2426)$ \\
\hline DHRS4 & Phase I & UCEC & cg05171021 & + & $(0.0695)$ & - & $(-0.3721)$ & nsig & $(-0.0753)$ & $(-0.2426)$ \\
\hline$R X R A$ & Modifier & HapMap & cg14468605 & + & $(0.1610)$ & - & $(-0.2844)$ & nsig & $(0.0263)$ & $(-0.2813)$ \\
\hline GSR & Phase I & BRCA & cg19913448 & + & $(0.0184)$ & nsig & $(-0.1450)$ & - & $(-0.2636)$ & $(-0.4871)$ \\
\hline TAP2 & Transporter & BRCA & $\operatorname{cg} 16951654$ & - & $(-0.0152)$ & - & $(-0.3080)$ & - & $(-0.1275)$ & $(0.4710)$ \\
\hline GSTCD & Phase II & BRCA & cg24545054 & + & $(0.0193)$ & nsig & $(-0.1692)$ & - & $(-0.1570)$ & $(-0.7471)$ \\
\hline GPX7 & Phase I & BRCA & $\operatorname{cg} 23272399$ & - & $(-0.0881)$ & - & $(-0.6346)$ & - & $(-0.5050)$ & $(0.2497)$ \\
\hline SLC7A5 & Transporter & BRCA & cg05834639 & - & $(-0.0799)$ & - & $(-0.5223)$ & - & $(-0.5008)$ & $(0.9730)$ \\
\hline SLC7A5 & Transporter & BRCA & $\operatorname{cg} 27560818$ & - & $(-0.1069)$ & - & $(-0.6634)$ & - & $(-0.3998)$ & $(0.9730)$ \\
\hline$A L D H 7 A 1$ & Phase I & HapMap & cg00997655 & + & $(0.1593)$ & nsig & $(0.1670)$ & + & $(0.5570)$ & (1.7172) \\
\hline ALDH3B2 & Phase I & HapMap & cg20333014 & + & $(0.1076)$ & + & $(0.3060)$ & nsig & $(0.0814)$ & (1.5288) \\
\hline ALDH3B2 & Phase I & HapMap & $\operatorname{cg} 24563501$ & + & $(0.1303)$ & + & $(0.3571)$ & nsig & $(-0.0426)$ & (1.5288) \\
\hline SLCO3A1 & Transporter & HapMap & cg07725123 & + & $(0.1683)$ & nsig & (0.1192) & + & $(0.3353)$ & (1.4612) \\
\hline$P O R$ & Modifier & COAD & cg13915028 & - & $(-0.1199)$ & nsig & $(-0.3238)$ & - & $(-0.2159)$ & (0.1099) \\
\hline$A B C C 6$ & Transporter & HapMap & cg01136471 & + & $(0.0912)$ & nsig & $(0.2155)$ & - & $(-0.3313)$ & $(-0.7201)$ \\
\hline GSTT2 & Phase II & HapMap & cg21667215 & + & $(0.0838)$ & nsig & $(-0.0752)$ & + & (0.3379) & (1.1582) \\
\hline$A B C C 12$ & Transporter & BRCA & cg26758427 & - & $(-0.0347)$ & nsig & $(0.0941)$ & + & $(0.0937)$ & $(-0.3496)$ \\
\hline SOD1 & Modifier & BRCA & cg19949530 & - & $(-0.0884)$ & - & $(-0.2237)$ & - & $(-0.2350)$ & $(0.2642)$ \\
\hline \multicolumn{11}{|c|}{ 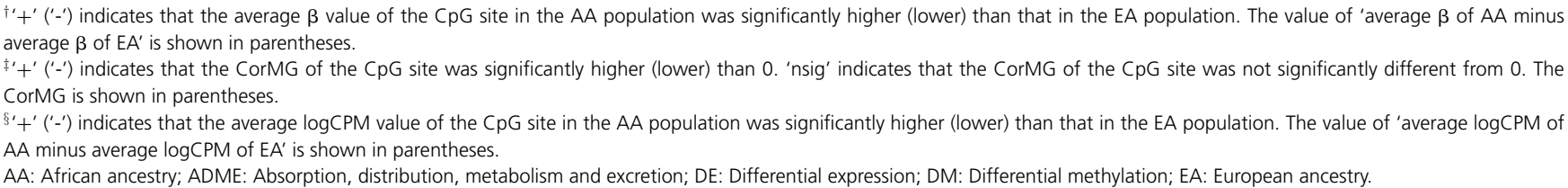 } \\
\hline
\end{tabular}

pModel $\mathrm{B}=9.86 \times 10^{-15}$, p podel $\mathrm{C}=8.32 \times 10^{-13}$, Table 3A). All of the four $(100 \%)$ analyzed DHRS7 promoter CpG sites (cg23283614, cg24461412, cg00846580 and cg18906360) were E-CpGs, and they all exhibited significantly higher average $\beta$ values in the AA population than that in the EA population (Table 3A). Two E-CpGs close to TSS (cg24461412 and cg00846580) showed significantly negative CorMG with DHRS7 expression in at least one population.

The other E-CpG cg01878807 was located in the promoter region of another Phase I metabolism-related gene, DHRS4. Similar to cg23283614, cg01878807 exhibited a significantly higher average $\beta$ value in the AA population than that in the EA population ( $\mathrm{p}_{\text {Model } \mathrm{A}}=1.93 \times 10^{-21}$, podel $\mathrm{B}=8.70 \times 10^{-27}$, p Model $\mathrm{C}=5.14 \times 10^{-21}$, Table 3B). Four of the six (67\%) analyzed DHRS4 promoter CpG sites were E-CpGs (cg01878807, cg01937701, $\operatorname{cg} 05171021$ and $\operatorname{cg} 18981338$ ), and they all exhibited higher average $\beta$ values in the AA population than that in the EA population (Table 3B). Moreover, these four promoter E-CpGs all showed significantly negative CorMG with DHRS4 expression in at least one population.

Straightforward relationships between methylation \& gene expression in ADME genes

For E-CpGs on ADME genes, to investigate the relationship between the interethnic methylation difference and the variation of gene expression given the significant CorMG, we performed DE analysis and verified whether a straightforward relationship exists between an E-CpG and the corresponding ADME gene (see Materials \& methods for details). We identified straightforward relationships between 8 (2, 0, 2 and 7) E-CpGs and their corresponding genes in the BRCA (COAD, HNSC, UCEC and HapMap) dataset (Table 4; Supplementary Table 5E), and 13 of all these E-CpGs showed the relationships through negative CorMG. Among these E-CpGs, the E-CpG cg27560818 in an intron region of the ADME gene $S L C 7 A 5$ showed the strongest CorMG (CorMG ${ }_{\mathrm{AA}}=-0.6634, \mathrm{p}<2.2 \times 10^{-16}$, Figure $3 \&$ Supplementary Table 5E) in the BRCA dataset. The straightforward relationship indicated that the observed lower average $\beta$ value in the AA population was related to the higher average SLC7A5 expression in the AA 


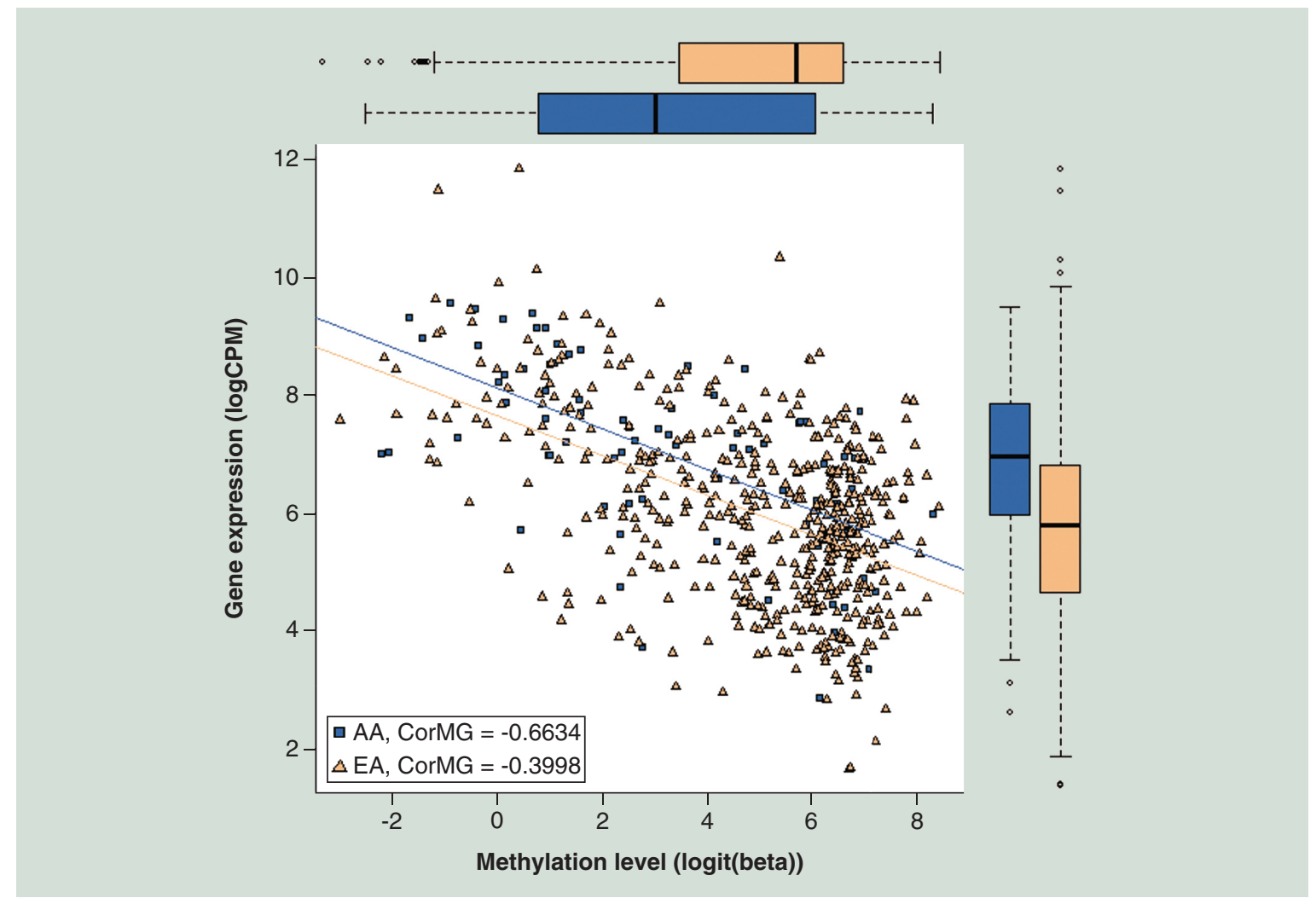

Figure 3. Straightforward relationship between E-CpG cg27560818 and the corresponding absorption, distribution, metabolism and excretion gene SLC7A5 in the BRCA dataset.

The findings indicate that the observed lower average $\beta$ value in the AA population (top panel) is related to the higher average SLC7A5 expression in the AA population (right panel) through negative CorMG in both studied populations (middle panel).

AA: African ancestry; ADME: Absorption, distribution, metabolism and excretion.

population through negative CorMG in both AA and EA populations. The findings may be a possible explanation for the differential tamoxifen treatment responses in the AA and EA populations (Supplementary Figure 7); the details are provided in Discussion. A similar straightforward relationship was observed for the E-CpG cg05834639, which was also located in an intron region of SLC7A5 (Supplementary Figure 8). No straightforward relationship between methylation and gene expression in MeDR was found.

Tumor-specific E-CpGs \& E-CpGs identified both in tumors \& LCL

To investigate the relationship between E-CpGs found in tumors and those found in LCLs, we compared E-CpGs in each dataset (Figure $4 \mathrm{~A}$ ) and verified whether the $\mathrm{CpGs}$ identified as E-CpGs in all cancer datasets are also E-CpGs in the HapMap dataset. A total of $80 \mathrm{CpGs}$ were identified as E-CpGs in all cancer datasets - 44 were also E-CpGs in HapMap dataset, 30 were included in the HapMap DM analysis but were not E-CpGs (i.e., tumor-specific E-CpGs shared by the four studied cancers), and 6 were not included in the HapMap DM analysis because of exclusion in the data QC procedure (Figure 4B).

Sixty-five of the 80 aforementioned E-CpGs were located in or close to some genes. Of these 65 E-CpGs, 13 showed significant CorMG (in at least one population) in all cancer datasets (Figure 4B and Supplementary Table 6). Of these, cg00669623 was the only $\mathrm{CpG}$ site that was identified as an E-CpG and showed significant CorMG in all cancer and HapMap datasets. The E-CpG cg00669623 was located in the promoter CGI region of CDK11A. Straightforward relationships were observed between cg00669623 and CDK11A in the BRCA and HapMap datasets; the higher average $\beta$ value of cg00669623 in the AA population was related to the higher average $C D K 11 A$ expression in the AA population through positive CorMG. A similar straightforward relationship was also observed between cg00669623 and gene $C D K 11 B$, which is adjacent to $C D K 11 A$, in the BRCA dataset. 


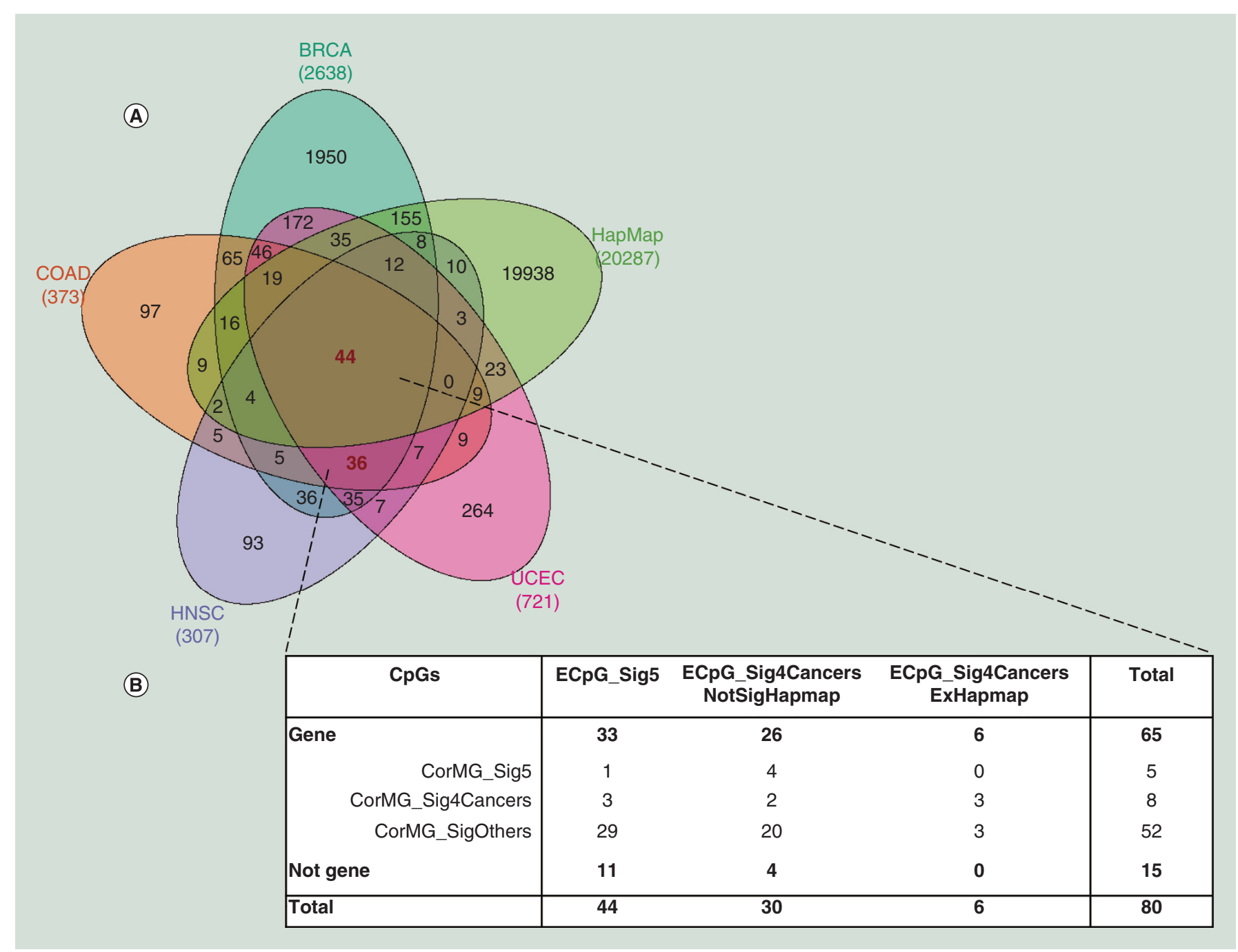

Figure 4. Comparison of E-CpGs in studied datasets.

(A) Venn diagram of E-CpGs in all studied datasets. (B) Distribution of $80 \mathrm{CpG}$ sites that are E-CpGs in all cancer datasets. Group 'ECpG_Sig5' indicates the CpG sites identified as E-CpGs in all five datasets. 'ECpG_Sig4Cancers_NotSigHapmap' indicates the CpG sites identified as E-CpGs in only four cancer datasets, and the $\mathrm{CpG}$ sites are not differentially methylated in the HapMap dataset.

'ECpG_Sig4Cancers_ExHapmap' indicates the CpG sites identified as E-CpGs in only four cancer datasets, and the CpG sites are excluded from DM analysis in the HapMap dataset. Group 'Gene' and 'Not gene' indicate the CpG sites close to genes and not close to genes, respectively. Group 'CorMG_Sig5' indicates the CpG sites with significant CorMG in all five datasets. 'CorMG_Sig4Cancers' indicates the CpG sites with significant CorMG in only four cancer datasets. 'CorMG_SigOthers' indicates CpG sites not included in groups 'CorMG_Sig5' and 'CorMG_Sig4Cancers'.

DM: Differential methylation.

We observed that three CpG sites (cg04286297, cg08477332 and cg24545054) were E-CpGs in all cancer and HapMap datasets. However, they showed significant CorMG with the corresponding genes (FAM103A1, S100A14 and INTS12) only in the cancer datasets. This finding implied that the role of these E-CpGs in gene regulation in LCLs may be different from that in gene regulation in tumor cells. Six CpG sites (cg01878807, cg04193820, cg05681977, cg20188490, cg12615916 and cg18392540) were tumor-specific E-CpGs shared by the four studied cancers, and they showed significant CorMG with the corresponding genes (DHRS4-AS1, RNF135, SLC39A4, PRH1, CDC42BPA and ANGEL2) in all four cancer datasets or all five studied datasets. These genes reflected the ethnic difference in tumors. 


\section{Discussion}

The results of this study demonstrated that DM between the AA and EA populations exists in MeDR and ADME genes in some tissue types and cancer types. Moreover, some of the identified E-CpGs are associated with the expression of the corresponding genes and linked to the drug response and ADME. The ethnic effect should be considered in pharmacoepigenomic studies. For example, when identifying DNA methylation markers to predict the drug response or evaluate the drug dosage, to reduce false positive results, population stratification or admixture should be considered in the study design and the consequent statistical analyses. If the predictive markers developed on the basis of samples from a single ethnic population are to be applied to other populations, an advanced investigation of transferability is required. In addition, comparison of E-CpGs across the datasets in this study revealed significant differences in global patterns and individual CpG sites between the cancer datasets and the HapMap dataset. These results highlight the complexity of epigenetic mechanisms and the importance of considering ethnic and tissue effects in the studies and applications of DNA methylation.

Our study provides clues about the differential responses and ADME of certain treatments between the AA and EA populations. For example, our findings suggest the role of the ADME gene SLC7A5, a solute carrier (SLC) transporter, in racial disparity in the tamoxifen treatment outcome of breast cancer patients - AA patients exhibited lower sensitivity to tamoxifen than did EA patients [41,42] (Supplementary Figure 7). Our BRCA dataset analysis showed that the E-CpG cg27560818 on SLC7A5 exhibited a significantly lower methylation level in the AA population than that in the EA population. Moreover, we observed that the E-CpG cg27560818 was negatively associated with the gene expression of SLC7A5; thus, the expression level of SLC7A5 was significantly higher in the AA population than that in the EA population. Previous studies have reported that breast cancer patients with higher SLC7A5 expression exhibited a poorer response to tamoxifen therapy [43]; this pattern is coincident with the reduced sensitivity to tamoxifen in AA patients. A recent study showed that the unfolded protein response (UPR) may be a cause for disparity in the tamoxifen treatment outcome between AA and EA women with breast cancer [44], and UPR genes were upregulated in the tumors of AA patients, and these patients showed lower sensitivity to tamoxifen than did EA patients. Our finding on SLC7A5 is also related to UPR, as demonstrated by a report showing that the expression of SLC7A5 increased significantly after endoplasmic reticulum stress, which induced UPR [45]. However, the hypothetical mechanism about that interethnic DNA methylation difference influences response to tamoxifen therapy in different populations is still incomplete until the straightforward relationship between methylation and gene expression of SLC7A5 gene is proved as a causal relationship.

In our results, some E-CpGs did not show a significant relationship to the gene expression of the corresponding genes (i.e., no significant CorMG, DE gene or straightforward relationship); for example, the DE results of the MeDR gene BRCA1 and the ADME gene DHRS7 were not significant. The three reasons that the methylation of these E-CpGs did not show a significant relationship to the expression of the corresponding genes are as follows: first, gene expression may be regulated by methylation not only in cis but also in trans [1]. Second, nonbiological issues, such as an insufficient number of samples, may limit the power to detect the relationship. Last, in addition to DNA methylation, other factors are involved in the regulation of gene expression, such as noncoding RNAs, genetic polymorphisms, structural variations, histone modifications and the chromatin state. An interesting example was found in the ADME gene DHRS4 in the BRCA dataset. We could not establish a straightforward relationship between the promoter E-CpGs (cg01878807 and cg05171021) and the gene expression of DHRS4. However, the two promoter E-CpGs displayed straightforward relationships between their higher average methylation levels and their lower average expression levels of long noncoding RNA DHRS4-AS1 through negative CorMG in the AA population (Supplementary Table 6D). DHRS4-AS1 induces the promoter methylation of DHRS4 and silences DHRS4 expression [46]. Therefore, our result reflects the previous finding and further shows its interethnic difference. In a previous study, the expression of DHRS4 was incorporated into gene sets for outcome prediction in breast cancer patients [47]; however, the ethnic effect was not accounted for in the outcome prediction, although it should be.

On the basis of the finding that not many E-CpGs in the studied datasets overlapped (Figure 4A), different E-CpG methylation patterns between the cancer datasets and the HapMap dataset may imply that the E-CpGs exhibit different functions in these two groups. For a brief understanding of the regulatory roles of E-CpGs, we used the BRCA and HapMap datasets as examples and performed enrichment analyses [48]. The result showed that in both datasets, their E-CpGs located close to the genes were overrepresented in the gene set involved in the ATP-delivering process of oxidative phosphorylation (Supplementary Table 7). Thus, some differences may exist 
in oxidative phosphorylation between the AA and EA populations. Notably, studies have reported direct functional coupling between oxidative phosphorylation and mitochondrial creatine kinase activity [49], and a previous study reported that black people have higher creatine kinase activity than white people in tissues with high and fluctuating energy demands [50]. For E-CpGs close to genes in the BRCA dataset, we found some additional enriched gene sets that were related to the cell cycle, such as genes regulated by $M Y C$, genes encoding cell cycle-related targets of E2F transcription factors, genes involved in the G2/M checkpoint and genes upregulated by the activation of the $\mathrm{PI} 3 \mathrm{~K} / \mathrm{AKT} / \mathrm{mTOR}$ pathway. The result fundamentally reflects the previous finding of the abnormal functioning of cell cycle components in AA women with breast cancer compared with their EA counterparts [51]; however, additional studies are required to understand the role of each E-CpG.

Samples in the HapMap dataset were LCLs; thus, their E-CpG methylation patterns might be affected by the variation introduced by Epstein-Barr virus transformation [52,53]. To address this issue, we extracted the differentially methylated CPG sites between the AA and EA populations in both blood and LCL samples from a previous study [54]. We investigated the methylation patterns of these ethnicity associated CpG sites by using the data adopted by that previous study (the data were downloaded from NCBI GEO: the accession number of LCL data [LCL_v] was GSE36369; the accession number of blood data [blood_v] was GSE36064). The results in both LCL_v and blood_v were similar to those in the HapMap dataset, in which low methylation in the promoter region and high methylation in the gene body and $3^{\prime}$-UTR regions were less obvious in the distribution of ethnicity associated CpG sites compared with methylation in the whole methylome (Supplementary Figures 9 \& 10). This finding implied the similarity of global methylation patterns between LCL and blood samples.

For the cancer datasets, we verified whether known cancer subtypes are confounders in our analysis. Patients' cancer subtype information was obtained from a data portal or previous publications of TCGA [21-24]. The results showed no significant differences in the proportion of each subtype between the AA and EA populations $\left(\mathrm{p}_{\mathrm{BRCA}}=0.7831, \mathrm{p}_{\mathrm{COAD}}=1, \mathrm{p}_{\mathrm{HNSC}}=0.6982\right.$, $\mathrm{pUCEC}=0.06657$, Supplementary Table 8$)$. Therefore, although we cannot completely exclude an impact of cancer subtype on our E-CpG analysis, this factor should not influence our findings. We also evaluated whether there was a cohort effect from the tissue source sites in our analysis. We examined the tissue source sites of those TCGA samples included in this study. The result showed that $>80 \%$ (1216 in 1500) of studied samples came from the institutions which collected more than one type of tumor samples (Supplementary Table 9). We cannot exclude potential cohort effect in our E-CpG analysis, but this factor should not influence our findings.

We investigated tumor-specific E-CpGs and the E-CpGs identified in both tumors and LCL (Supplementary Table 6). Many genes related to tumor-specific E-CpGs, such as ANGEL2 [55], CDC42BPA [56], RNF135 [57] and $S L C 39 A 4$ [58], have been reported to be involved in tumor progression and metastasis, and most of the genes are also associated with cell cycle regulation $[55,57,58]$. Our study not only provides results that are consistent with the previous finding that ethnic differences exist in cell cycle components in breast cancer patients [51], but also provides a new finding on the interethnic difference in tumor progression and metastasis. The gene CDK11A containing an E-CpG (i.e., cg00669623) was identified in all studied datasets. Previous studies have also discussed its role in tumor development and treatment [59,60]. CDK11A modulates autophagy [61], and autophagy plays a pivotal role in skin color determination [62], which is highly related to ethnicity. This finding may be helpful to explain why the CpG cg00669623 was identified as an E-CpG in both tumors and LCL.

Recently, on the basis of LCLs across the three groups of Americans with Caucasian, African or Han Chinese ancestry, Heyn et al. reported that two drug response genes, $A B C B 11$ and SPATC1L, contained 1 and 7 differentially methylated CpG sites, respectively, in the AA and EA populations [54]. We investigated our results according to their findings. We first analyzed these CpG sites in the HapMap dataset, which also used LCL samples (Supplementary Table 10). For $A B C B 11$, although the previously reported ethnicity-associated site (i.e., cg18912768) was not an E-CpG in our study, Heyn et al. and our study consistently showed a higher average $\beta$ value in the AA population than in the EA population $(0.58>0.42)$. Moreover, we found that an E-CpG cg00295325 on $A B C B 11$ was close to cg18912768 (distance < 100 base pairs) and was highly correlated with cg18912768 (Spearman's rank correlation coefficient $[\mathrm{r}]=0.64)$, although no significant relationship was observed between methylation and gene expression of $A B C B 11$ for both CpG sites. For SPATC1L, five of the seven reported CpG sites were E-CpGs, which further demonstrated straightforward relationships in our study. Heyn et al. reported that differentially methylated CpG sites at the TSS regulated gene expression differently compared with that by sites at the transcription end site [54], which was also found in this study. Furthermore, we analyzed $\mathrm{CpG}$ sites at these two drug response genes in the four cancer datasets. A CpG site (i.e., cg08742575) at the TSS of SPATC1L was identified as an E-CpG in the 
BRCA dataset, and it also showed a straightforward relationship, which was similar to the relationship found in the same CpG site in the HapMap dataset. Taken together, this investigation showed that our results are mostly consistent with previous findings, and our study further extended some previous findings in LCL samples to other tissue types. However, this investigation also reflected the limitation of our study that the list of genes related to the drug response is still incomplete because SPATC1L was not included in either MeDR or ADME genes. Only limited data can be accessed for a partial validation in this study. Until further replication, the current results should be interpreted carefully.

We also examined genetic basis of E-CpGs that were identified based on HapMap LCLs of EA and AA populations in this study. We found the association between SNPs and E-CpGs in the previous methylation quantitative trait locus studies. Zhang et al. [63] identified the corresponding methylation quantitative trait locus of 5240 (7306) CpGs based on HapMap LCLs of EA (AA) population, and 390 (495) of which CpGs were also E-CpGs in our study, where 3 (9) of which E-CpGs were located on ADME genes (Supplementary Table 11).

In this study, we illustrated an ethnic effect on DNA methylation difference, and the interethnic DNA methylation difference was further examined in relation to the treatment response and drug ADME in cancer patients. Ethnic effect may also influence lifestyle and environmental exposures in epigenetic epidemiology [64] and the CGI methylator phenotype (CIMP) [65]. CIMP, highly relevant to carcinogenesis and clinical outcomes, provides a useful diagnostic and prognostic molecular signature for cancers [65]. Previous studies observed that CIMP prevalence varied across ethnic populations [66]. Therefore, we also evaluated the interethnic methylation difference in the CIMP-associated CpG sites (i.e., the CpG sites which are significantly hypermethylated in the CIMP-tumors compared with the non-CIMP-tumors). Previous studies reported 2333 CIMP-associated CpG sites for breast cancer [67] and 488 CIMP-associated CpG sites for colorectal cancer [68], where 2092 (in 2333) and 462 (in 488) CpG sites can be found in the BRCA and COAD dataset from TCGA, respectively. Of which CIMP-associated CpG sites, 18 (1) exhibited a statistically significant interethnic effect in the BRCA (COAD) dataset after Bonferroni correction (Supplementary Table 12). The results reveal a clue for the ethnic effect on the CIMP-associated CpG sites.

Molecular pathological epidemiology (MPE), which combines traditional epidemiology and traditional molecular pathology, discusses how a specific molecular change is influenced by or interacted with lifestyle and environmental exposures to influence disease risk or treatment response [69-71]. This study focuses on the epigenetic changes in cancer, and it has been shown that some risk factors of cancers influence epigenetic marks [64]. For example, aging is a crucial risk factor for cancer [72,73] and also relevant to the treatment outcome [74]. Studies have shown that the epigenetic age of human tissues can be defined by the methylation level of $353 \mathrm{CpG}$ sites, which are termed clock CpGs [75], and that the epigenetic aging rates are significantly associated with ethnicity [76]. Here we discuss whether $\mathrm{CpG}$ sites related to the drug response or ADME would be affected by both the ethnic difference and the epigenetic age difference. We first verified whether the clock $\mathrm{CpGs}$ are located in or close to the MeDR/ADME genes in this study, and 9 such CpG sites were identified, of which 3 (6) of them showed a positive (negative) correlation with age (Supplementary Table 13). Among the 9 clock CpG sites, 2 (0,3,0 and 2) showed a statistically significant interethnic effect in the BRCA (COAD, HNSC, UCEC and HapMap) datasets after Bonferroni correction. Notably, all the significant clock E-CpGs showed a higher average $\beta$ value in the AA population than that in the EA population. Although future investigation is required for a comprehensive understanding of the interplay between ethnicity and age, we did find some CpG sites related to the drug response that showed DM levels by both ethnicity and age.

\section{Conclusion}

In this study, we performed the first systematic study to examine the population differentiation effect of DNA methylation on the treatment response and drug ADME. We identified E-CpG sites and found that some of them were related to the drug response and ADME. We also found clues that some of the E-CpG sites may trigger differential gene expression and influence the drug response and ADME in different populations. Our findings demonstrated that the ethnic effects on pharmacoepigenetics and the patterns may change according to tissue types. Applying the right treatment and right dosage to the patients from the ethnic population is critical in clinical practice and pharmacoepigenetics research. 
Supplementary data

To view the supplementary data that accompany this paper please visit the journal website at: www.futuremedicine.com/doi/full/10.2217/epi-2017-0046

\section{Acknowledgements}

The results published here are in part based upon data generated by TCGA Research Network: http://cancergenome.nih.gov/. Part of the material is based on the PhD thesis of the first author (S-K Chu) under the supervision of the last author ( $\mathrm{H}-\mathrm{C}$ Yang). The authors sincerely thank four anonymous reviewers for their very constructive and insightful comments that helped in preparing our manuscript.

Financial \& competing interests disclosure

This work was partially supported by the Ministry of Science and Technology of Taiwan under Grant MOST 106-2314-B-001003-MY3. The authors have no other relevant affiliations or financial involvement with any organization or entity with a financial interest in or financial conflict with the subject matter or materials discussed in the manuscript apart from those disclosed.

No writing assistance was utilized in the production of this manuscript.

Open access

This work is licensed under the Attribution-NonCommercial-NoDerivatives 4.0 Unported License. To view a copy of this license, visit http://creativecommons.org/licenses/by-nc-nd/4.0/

\section{Summary points}

- DNA methylation, in which methyl groups are added to the C-5 position of the cytosine ring in the CpG dinucleotide of DNA, is a major epigenomic mechanism.

- In contrast to genomic mechanisms, DNA methylation influences phenotypes (e.g., drug response and absorption, distribution, metabolism and excretion [ADME]) without any involvement of the DNA sequence change.

- Ethnic differences have been found in DNA methylation; however, the influence of interethnic DNA methylation differences on the drug response and ADME remains largely unknown.

- We identified E-CPG sites and found that some of them were related to the drug response and ADME.

- We found clues that some of the E-CpG sites may trigger differential gene expression and influence the drug response and $A D M E$ in different populations.

- The results of cg27560818 on SLC7A5 were combined with the finding of genomic studies to explain the poor response to tamoxifen therapy in African ancestry patients.

- Our findings demonstrated that the ethnic effects on pharmacoepigenetics and the patterns may change according to tissue types.

- Applying the right treatment and right dosage to the patients from the ethnic population is critical in clinical practice and pharmacoepigenetics research.

\section{References}

1 Portela A, Esteller M. Epigenetic modifications and human disease. Nat. Biotechnol. 28(10), 1057-1068 (2010).

2 Weber M, Schubeler D. Genomic patterns of DNA methylation: targets and function of an epigenetic mark. Curr. Opin. Cell Biol. 19(3), 273-280 (2007).

3 Lister R, Pelizzola M, Dowen RH et al. Human DNA methylomes at base resolution show widespread epigenomic differences. Nature 462(7271), 315-322 (2009).

4 Jones PA. Functions of DNA methylation: islands, start sites, gene bodies and beyond. Nat. Rev. Genet. 13(7), 484-492 (2012).

5 Lechner M, Boshoff C, Beck S. Cancer epigenome. Adv. Genet. 70, 247-276 (2010).

6 Gomez A, Ingelman-Sundberg M. Pharmacoepigenetics: its role in interindividual differences in drug response. Clin. Pharmacol. Ther. 85(4), 426-430 (2009).

7 Heyn H, Esteller M. DNA methylation profiling in the clinic: applications and challenges. Nat. Rev. Genet. 13(10), 679-692 (2012).

8 Winter S, Fisel P, Buttner F et al. Methylomes of renal cell lines and tumors or metastases differ significantly with impact on pharmacogenes. Sci. Rep. 6, 29930 (2016).

9 Esteller M, Garcia-Foncillas J, Andion E et al. Inactivation of the DNA-repair gene MGMT and the clinical response of gliomas to alkylating agents. N. Engl. J. Med. 343(19), 1350-1354 (2000).

10 Wiencke JK. Impact of race/ethnicity on molecular pathways in human cancer. Nat. Rev. Cancer 4(1), 79-84 (2004). 


\section{Research Article Chu \& Yang}

11 Kurian AK, Cardarelli KM. Racial and ethnic differences in cardiovascular disease risk factors: a systematic review. Ethn. Dis. 17(1), 143-152 (2007).

12 Caprio S, Daniels SR, Drewnowski A et al. Influence of race, ethnicity, and culture on childhood obesity: implications for prevention and treatment: a consensus statement of Shaping America's Health and the Obesity Society. Diabetes Care 31(11), 2211-2221 (2008)

13 Chung WH, Hung SI, Hong HS et al. Medical genetics: a marker for Stevens-Johnson syndrome. Nature 428(6982), 486 (2004).

14 Maisano Delser P, Fuselli S. Human loci involved in drug biotransformation: worldwide genetic variation, population structure, and pharmacogenetic implications. Hum. Genet. 132(5), 563-577 (2013).

15 Ramos E, Doumatey A, Elkahloun AG et al. Pharmacogenomics, ancestry and clinical decision-making for global populations. Pharmacogenomics J. 14(3), 217-222 (2014).

16 Fraser HB, Lam LL, Neumann SM, Kobor MS. Population-specificity of human DNA methylation. Genome Biol. 13(2), R8 (2012).

17 Moen EL, Zhang X, Mu W et al. Genome-wide variation of cytosine modifications between European and African populations and the implications for complex traits. Genetics 194(4), 987-996 (2013).

18 Xia YY, Ding YB, Liu XQ et al. Racial/ethnic disparities in human DNA methylation. Biochim. Biophys. Acta 1846(1), 258-262 (2014).

19 Conway K, Edmiston SN, Tse CK et al. Racial variation in breast tumor promoter methylation in the Carolina Breast Cancer Study. Cancer Epidemiol. Biomarkers Prev. 24(6), 921-930 (2015).

20 Lokk K, Modhukur V, Rajashekar B et al. DNA methylome profiling of human tissues identifies global and tissue-specific methylation patterns. Genome Biol. 15(4), r54 (2014).

21 Cancer Genome Atlas N. Comprehensive molecular characterization of human colon and rectal cancer. Nature 487(7407), 330-337 (2012).

22 Cancer Genome Atlas N. Comprehensive molecular portraits of human breast tumours. Nature 490(7418), 61-70 (2012).

23 Cancer Genome Atlas Research Network, Kandoth C, Schultz N et al. Integrated genomic characterization of endometrial carcinoma. Nature 497(7447), 67-73 (2013).

24 Cancer Genome Atlas N. Comprehensive genomic characterization of head and neck squamous cell carcinomas. Nature 517(7536), 576-582 (2015)

25 Dedeurwaerder S, Defrance M, Calonne E, Denis H, Sotiriou C, Fuks F. Evaluation of the infinium methylation 450K technology. Epigenomics 3(6), 771-784 (2011).

26 Sandoval J, Heyn H, Moran S et al. Validation of a DNA methylation microarray for 450,000 CpG sites in the human genome. Epigenetics 6(6), 692-702 (2011).

27 Wang K, Singh D, Zeng Z et al. MapSplice: accurate mapping of RNA-seq reads for splice junction discovery. Nucleic Acids Res. 38(18), e178 (2010).

28 UNC Bioinformatics Utilities. https://github.com/mozack/ubu

29 Li B, Dewey CN. RSEM: accurate transcript quantification from RNA-Seq data with or without a reference genome. $B M C$ Bioinformatics 12, 323 (2011).

30 Lappalainen T, Sammeth M, Friedlander MR et al. Transcriptome and genome sequencing uncovers functional variation in humans. Nature 501(7468), 506-511 (2013).

31 T Hoen PA, Friedlander MR, Almlof $\mathrm{J}$ et al. Reproducibility of high-throughput mRNA and small RNA sequencing across laboratories. Nat. Biotechnol. 31(11), 1015-1022 (2013).

32 Marco-Sola S, Sammeth M, Guigo R, Ribeca P. The GEM mapper: fast, accurate and versatile alignment by filtration. Nat. Methods 9(12), 1185-1188 (2012).

33 Griebel T, Zacher B, Ribeca P et al. Modelling and simulating generic RNA-Seq experiments with the flux simulator. Nucleic Acids Res. 40(20), 10073-10083 (2012).

34 Teschendorff AE, Marabita F, Lechner $\mathrm{M}$ et al. A beta-mixture quantile normalization method for correcting probe design bias in illumina infinium 450 k DNA methylation data. Bioinformatics 29(2), 189-196 (2013).

35 Johnson WE, Li C, Rabinovic A. Adjusting batch effects in microarray expression data using empirical Bayes' methods. Biostatistics 8(1), 118-127 (2007).

36 Leek JT, Storey JD. Capturing heterogeneity in gene expression studies by surrogate variable analysis. PLoS Genetics 3(9), 1724-1735 (2007).

37 Du P, Zhang X, Huang CC et al. Comparison of beta-value and M-value methods for quantifying methylation levels by microarray analysis. BMC Bioinformatics 11, 587 (2010).

38 Miller RG. Simultaneous Statistical Inference (2nd Edition). Springer-Verlag, NY, USA (1981).

39 Hartmann O, Spyratos F, Harbeck N et al. DNA methylation markers predict outcome in node-positive, estrogen receptor-positive breast cancer with adjuvant anthracycline-based chemotherapy. Clin. Cancer Res. 15(1), 315-323 (2009). 
40 Veeck J, Ropero S, Setien F et al. BRCA1 CpG island hypermethylation predicts sensitivity to poly(adenosine diphosphate)-ribose polymerase inhibitors. J. Clin. Oncol. 28(29), e563-e564; author reply e565-e566 (2010).

41 Wright JL, Reis IM, Zhao W et al. Racial disparity in estrogen receptor positive breast cancer patients receiving trimodality therapy. Breast 21(3), 276-283 (2012).

42 Tichy JR, Deal AM, Anders CK, Reeder-Hayes K, Carey LA. Race, response to chemotherapy and outcome within clinical breast cancer subtypes. Breast Cancer Res. Treat 150(3), 667-674 (2015).

43 Mihaly Z, Kormos M, Lanczky A et al. A meta-analysis of gene expression-based biomarkers predicting outcome after tamoxifen treatment in breast cancer. Breast Cancer Res. Tr. 140(2), 219-232 (2013).

44 Eltayeb AE, Demas DM, Clarke R, Shajahan-Haq AN. The unfolded protein response may contribute to racial disparity in endocrine responsiveness in breast cancer. Cancer Res. 1(3), 640-644 (2015).

45 Dombroski BA, Nayak RR, Ewens KG, Ankener W, Cheung VG, Spielman RS. Gene expression and genetic variation in response to endoplasmic reticulum stress in human cells. Am. J. Hum. Genet. 86(5), 719-729 (2010).

$46 \mathrm{Li}$ Q, Su Z, Xu X et al. AS1DHRS4, a head-to-head natural antisense transcript, silences the DHRS4 gene cluster in cis and trans. Proc. Natl Acad. Sci. USA 109(35), 14110-14115 (2012).

47 Korkola JE, Blaveri E, Devries $S$ et al. Identification of a robust gene signature that predicts breast cancer outcome in independent data sets. BMC Cancer 7, 61 (2007).

48 Subramanian A, Tamayo P, Mootha VK et al. Gene set enrichment analysis: a knowledge-based approach for interpreting genome-wide expression profiles. Proc. Natl Acad. Sci. USA 102(43), 15545-15550 (2005).

49 Schlattner U, Tokarska-Schlattner M, Wallimann T. Mitochondrial creatine kinase in human health and disease. BBA Mol. Basis Dis. 1762(2), 164-180 (2006).

50 Brewster LM, Coronel CMD, Sluiter W, Clark JF, Van Montfrans GA. Ethnic differences in tissue creatine kinase activity: an observational study. PLoS ONE 7(3), e32471 (2012).

51 Danforth DN. Disparities in breast cancer outcomes between Caucasian and African American women: a model for describing the relationship of biological and nonbiological factors. Breast Cancer Res. 15(3), 208 (2013).

52 Grafodatskaya D, Choufani S, Ferreira JC et al. EBV transformation and cell culturing destabilizes DNA methylation in human lymphoblastoid cell lines. Genomics 95(2), 73-83 (2010).

53 Caliskan M, Cusanovich DA, Ober C, Gilad Y. The effects of EBV transformation on gene expression levels and methylation profiles. Hum. Mol. Genet. 20(8), 1643-1652 (2011).

54 Heyn H, Moran S, Hernando-Herraez I et al. DNA methylation contributes to natural human variation. Genome Res. 23(9), 1363-1372 (2013).

55 Yi X, Hong M, Gui B et al. RNA Processing and modification protein, carbon catabolite Repression 4 (Ccr4), arrests the cell cycle through p21-dependent and p53-independent pathway. J. Biol. Chem. 287(25), 21045-21057 (2012).

56 Wilkinson S, Paterson HF, Marshall CJ. Cdc42-MRCK and Rho-ROCK signalling cooperate in myosin phosphorylation and cell invasion. Nat. Cell Biol. 7(3), U255-U245 (2005).

57 Liu YJ, Wang F, Liu YS et al. RNF135, RING finger protein, promotes the proliferation of human glioblastoma cells in vivo and in vitro via the ERK pathway. Sci. Rep. 6, 20642 (2016).

58 Li M, Zhang YQ, Liu ZJ et al. Aberrant expression of zinc transporter ZIP4 (SLC39A4) significantly contributes to human pancreatic cancer pathogenesis and progression. Proc. Natl Acad. Sci. USA 104(47), 18636-18641 (2007).

59 Lapenna S, Giordano A. Cell cycle kinases as therapeutic targets for cancer. Nat. Rev. Drug Discov. 8(7), 547-566 (2009).

60 Malumbres M, Barbacid M. Cell cycle, CDKs and cancer: a changing paradigm. Nat. Rev. Cancer 9(3), 153-166 (2009).

61 Wilkinson S, Croft DR, O'prey J et al. The cyclin-dependent kinase PITSLRE/CDK11 is required for successful autophagy. Autophagy 7(11), 1295-1301 (2011).

62 Murase D, Hachiya A, Takano K et al. Autophagy has a significant role in determining skin color by regulating melanosome degradation in keratinocytes. J. Invest. Dermatol. 133(10), 2416-2424 (2013).

63 Zhang X, Moen EL, Liu C et al. Linking the genetic architecture of cytosine modifications with human complex traits. Hum. Mol. Genet. 23(22), 5893-5905 (2014).

64 Barrow TM, Michels KB. Epigenetic epidemiology of cancer. Biochem. Biophys. Res. Commun. 455(1-2), 70-83 (2014).

65 Suzuki H, Yamamoto E, Maruyama R, Niinuma T, Kai M. Biological significance of the CpG island methylator phenotype. Biochem. Biophys. Res. Commun. 455(1-2), 35-42 (2014).

66 Weisenberger DJ, Levine AJ, Long TI et al. Association of the colorectal CpG island methylator phenotype with molecular features, risk factors, and family history. Cancer Epidemiol. Biomarkers Prev. 24(3), 512-519 (2015).

67 Fang F, Turcan S, Rimner A et al. Breast cancer methylomes establish an epigenomic foundation for metastasis. Sci. Transl. Med. 3(75), 75ra25 (2011). 
68 Hinoue T, Weisenberger DJ, Lange CP et al. Genome-scale analysis of aberrant DNA methylation in colorectal cancer. Genome Res. 22(2), 271-282 (2012).

69 Ogino S, Chan AT, Fuchs CS, Giovannucci E. Molecular pathological epidemiology of colorectal neoplasia: an emerging transdisciplinary and interdisciplinary field. Gut 60(3), 397-411 (2011).

70 Ogino S, Lochhead P, Chan AT et al. Molecular pathological epidemiology of epigenetics: emerging integrative science to analyze environment, host, and disease. Mod. Pathol. 26(4), 465-484 (2013).

71 Ogino S, Nishihara R, Vanderweele TJ et al. The role of molecular pathological epidemiology in the study of neoplastic and non-neoplastic diseases in the era of precision medicine. Epidemiology 27(4), 602-611 (2016).

72 Anisimov VN, Sikora E, Pawelec G. Relationships between cancer and aging: a multilevel approach. Biogerontology 10(4), 323-338 (2009).

73 De Magalhaes JP. How ageing processes influence cancer. Nat. Rev. Cancer 13(5), 357-365 (2013).

74 Mangoni AA, Jackson SH. Age-related changes in pharmacokinetics and pharmacodynamics: basic principles and practical applications. Br. J. Clin. Pharmacol. 57(1), 6-14 (2004).

75 Horvath S. DNA methylation age of human tissues and cell types. Genome Biol. 14(10), R115 (2013).

76 Horvath S, Gurven M, Levine ME et al. An epigenetic clock analysis of race/ethnicity, sex, and coronary heart disease. Genome Biol. 17(1), 171 (2016). 